544. J. Houben und A. Schottmüller:

Synthese aromatischer Amidosäuren durch Umlagerung. II.

[Aus dem Chemischen Institut der Universitit Berliu.]

(Eiogegangen an 2. Oktober 1909.)

Vor einigen Jahren ${ }^{1}$ ) hat der eine von uns in einer kurzen vorläufigen Mitteilung über Versuche berichtet, durch Übertragung der Kolbe schen Salicylsiure-Synthese auf stickstof haltige Benzol-Ahkömmlinge sekundïre Arylamine in Salze alkylierter Aninobenzoesäuren iiberzufïhren. Jiese Versuche liefen darauf hinaus, an einem phenylierten Stickstoffatom haftendes Carboxyl in den aromatischen Kern umzulageru.

Nach Seifert") lïßt sich das Stickistoffatom des Acetanilids carboxylieren, wenn man sein Natriumsalz, das leicht ans Acetanilid und alkoholischer Natronlauge gewonnen wird, nit Kohlensïure behandelt. Beim Erhitzen des so gebildeten phenylacetylcarbaminsauren Natriums gewaun Seifert indessen kein Aminobenzoat, vielmehr malonanilsaures Natrium.

Bei der Wiederholung des Seifertschen Versuchs unter den verschiedensteu Bedingungen gelang es ebenfalls nicht, auch nur die geringste Menge einer Amidosäure zu erzielen. Auch als an Stelle des Acetanilids Formanilid genommen wurde, entsprach das Realstionsprodukt dem von Sejfert isolierten: es entstand oxanilsaures Natrium.

Un die Wanderung des Carboxyls in einen aromatischen Kern zu erzwingen, gingen wir daher vom Benzanilidnatrium aus. Das hieraus entstehende phenylbenzoylcarhaminsaure Natrium mulite die COONaGruppe, falls überhaupt eive Wanderung eintrat, entwerler an den Anilinrest oder an das Benzoyl abgeben. Im ersten Falle sollte, Wanderung in Orthostellung vorausgesetzt, o-lienzoylamidobenzoesaures, im zweiten Falle phthalanilsaures Natrium entstehen:

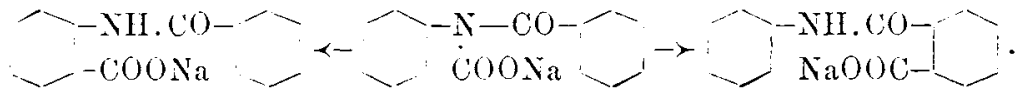

ther anch hier gelang es bis jetzt nicht, einen der beiden Körper zu fassen. Die angelagerte Kohlensüure wurde vielmehr anscheinend direkt wieder abgespalten. Es mulB unentschieden gelassen werden, ob dies auf die Anwesenbeit geringer Mengen Feuchtigkeit zuriickzufübren uder auch dem absolut trocknen Material eigentümlich ist. Im Verein nit überschiissiger Kohlensäure, welche bei den Versuchen nicht zu vermeiden war, wrkt wenig Wasser jedenfalls schon außerordentlich zersetzend.

1) Tiene Bericlite 37, 3978 [1904]. 3) Diese Berichte 18, 1358 [1885]. 
Diese Miberiolge füluten schließlich trotz der Augaben vou Titherley') zu Versuchen, die Arylamiue selbst direkt in ibre Natriumderivate und diese lavn in Carbaminate umzuwandelu. Es gelang nach verschiedenen Anläufen auch, sowohl Anilin wie Methylanilin in ihre Natriumverbindungen iiberzuführen. Nazu wurde einmal die Umsetzung des Amius mit Natriumamid,

$$
\mathrm{C}_{6} \mathrm{H}_{5} \cdot \mathrm{NH}_{2}+\mathrm{NH}_{3} \mathrm{Na}=\mathrm{C}_{6} \mathrm{H}_{5} \cdot \mathrm{NH} \cdot \mathrm{Na}+\mathrm{NH}_{3},
$$

und weiterbin ein dem H. Belartschen ") ähnliches Verfahren benutzt, wonach man das Natrium anf das siedende Arylamin unter intensivem Rühreu einwirken läßt. Eine Patentanmeldurg der Baseler chemischen Fabrik ${ }^{3}$ ) bringt mit gutem Frfolge Gemisebe von Alkalibydrat und Natrium bei einer Temperatur oberhalb $200^{\circ}$ zu Eiuwirkung auf das Arylanin. Beide Verfahren warer zur Zeit der Vornahme obiger Versuche nicht bekannt.

Wie die Untersuchung ergab, nehmen die Arylaminnatriumverbindungen Koalendioxyd auf. Es gelang aber nicht, durch Erhitzen Salze von Amidosäuren zu gewinnen. Auscheinend wird die Zersetzungstemperatur lange vor der Umlagerungstemperctur erreicht.

In neuerer Zeit hat Siegfried ${ }^{4}$ ) eiu Verfahren angegebeu, aus Aminosäuren in wäßriger Lijsung Salze von Carbonsäuren zu gewiunen. Dieses Verfabren kounte aber hier nicht in Betracht kommen. Auf interessante Mitteilungen von E. Mohri) muß indessen näber eingegangen werden.

F. Mohr zeigte, daß sich aus Phenylisocyanat und Barytwasser ein ganz beständiges Bariumsalz der Phenylcarbamiusäure herstellen läßt, das, gegen Wasser allein wenig empfindlich, durch wäßriges Kohlendioxyd völlig zersetzt wird. E. Mobr stellte dafür folgende Gleichung auf:

$\left(\mathrm{C}_{6} \mathrm{H}_{5} \cdot \mathrm{NH} . \mathrm{COO}\right)_{2} \mathrm{Ba}+\mathrm{CO}_{2}+\mathrm{H}_{2} \mathrm{O}=\mathrm{CO}_{3} \mathrm{Ba}_{4}+2 \mathrm{C}_{6} \mathrm{H}_{5} . \mathrm{NH}_{2}+2 \mathrm{CO}_{9}$.

Da hiervach bei dem durch Kohlensüure bewirkten Zerfall des Carbaminats die doppelte Menge der zersetzendev Kohlensäure als Zerfallsprodukt auftritt, so sollte theoretisch eire Molekel Kohlensäure genügen, um beliebig große Mengen des carbaminsauren Salzes zu zersetzen. Diese Zersetzung tritt aber nur bei Gegenwart von Wasser ein. Daraus ergibt sich, daß bei den Versuchen, Arylcarbaminate

1) Journ. Chem. Soc. 71, 462 [1897].

2) Patentanmoldung B. 41923 IV 12 q (ausgel. an 2. 5. 1907).

3) Patentanmelelung B. $42760 \mathrm{KV} 12$ \& (anigel. am 7. 1. 1907).

4) Ztschr. i. physiul. Chem. 44, 85 [1905]. Patentameldung S. 22164 IV 12 q (ausgel. am 4. 4. 1907). Ztschr. f. physiol. Chem. 46, 412; 54, 423, 437.

5) Journ. f. prakt. Chem. [2] 72, 297; 73, 177 und 2 (7?. 
umzulagern, das Wasser peinlich vou der Peaktionsmasse fernzuhalten war.

Wie schon in der kurzen vorläufigen Mitteilung ron J. Houben ${ }^{1}$ ) angegeben, gelang es schließlich, auf einfache Weise absolut wasserfreie und dabei zur Umlagerung sehr geeignete Carbaminate bei gewöhnlicher Temperatur, jai bei Eiskälte darzustellen. Das Verfahreu berubt auf den von L. Meunier ${ }^{2}$ ) zuerst beschriebenen Verhalten der Amine, mit Alkylmagnesiumbaloiden in Aminomagnesiumbaloide der allgemeinen Formel R.NH.Mg.Jal iberzugehen. Die ron Meunier herruhrende Gleichung

$$
\mathrm{R} . \mathrm{NH}_{2}+\mathrm{R}^{1} \cdot \mathrm{Mg} \cdot \mathrm{Hal}=\mathrm{R}^{1} \cdot \mathrm{NH} \cdot \mathrm{Mg} \cdot \mathrm{Hal}+\mathrm{R} \cdot \mathrm{H}
$$

gilt aber nur in dem Fall, daß äjuimolekulare Mengen des Amins und der Organomagnesiumverbindung zusanmentreffen oder das Amin in Überschuß ist. Belindet sich die Organomagnesiumverbindung im Überschuß, so vermag sie die dem Überschuß äruivalente Menge Aninomagnesiumhaloid zu binden und in ein Produlit bedeutend verminderter Realitivität überzugehen. Anders Jassen sich wenigstens die beim Anilin gemachten Beobachtungen ${ }^{3}$ ) nicht deuten. Sekundäre Aniline reagieren indessen nach inzwischen gemachten Beobachtungen ganz in der einfachen, der obigen Gleichung entsprechenden Weise, sofern sie nicht modilizierende Substituenten, wie etwa Oxalkyle oder Dialkylamin reste, enthalten.

Da sich in jedem Fall die Bildung der wenig reaktiven Joppelverbindung also leicht vermeiden läßt, vermag man durch Addition von Koblendioxyd an die Aninomagnesiumbaloide glatt $z u$ den gesuchten Carbaminaten zu gelangen $\left.{ }^{4}\right)$ :

$$
\text { R.NH. } \mathrm{Hg} . \mathrm{Hal}+\mathrm{CO}_{2}=\mathrm{R} . \mathrm{NH} . \mathrm{CO}_{3} \mathrm{Mg} \text {.Hal. }
$$

Wie in der zitierten vorläufigen Mitteiluug augegeben, beschränkte man sich auf die sekuudären Arylalkylanine, da die primären, wir vorausgesehen, kein Umlagerungsprodukt ergaben, vielmehr, eivem starken Isocyanatgeruch nach, eine avdere Zersetzung zu frleiden schieneu:

$$
\text { R.NH.COO Mg Hal = R.N:CO + HO. Mg.Hal. }
$$

Bei unseren späteren Versuchen - die allerdings meistens unter erheblich anderen Bedingungen vorgenommen wurden - kounten wir ein Isocyanat nicht mehr wahrnehmen, geschweige denn isolieren. Wir schreiben daher die zuerst beobachtete Isocyanatbildung einer

I) 1)ise Berichte 37, 3978 [1904].

3) Compt. rent. 136, 758; Bull. soe. chim. [3] 29, 314.

3) Houben, dieve Berichte 38, 3017 [1905].

4) Verol. Houben, diese Berichte 37, 3978 [1904]. 
Zufälligkeit zu, hesonders atuch, weil uns eine Spaltung ron Phenylcarbaminat in Base und Phenylisocyanat sehr unwahrscheinlich ist. Daß ein Phenylcarbaminat in wäBriger Lösung eine solche Spaltung nicht erleidet, hat E. Mohr nachgewiesen.

lix Grund für die negativen Liggebnisse, die wir lei Auwendung primärer drylamine erhielten, ist also wohl in der Bildung anderer Tebenprodukte zu suchen. Als solcbe kïnnen - in oft recht beträchtlicher Menge - Marustoffe autreten, deren Entstrhung sich wohl an besten in folgender Weise erlilïrt:

$2 \mathrm{C}_{6} \mathrm{H}_{5} . \mathrm{NHl} . \mathrm{COONg} . \mathrm{Hal}=\mathrm{C}_{6} \mathrm{H}_{5} . \mathrm{NH} . \mathrm{CO} . \mathrm{NH} . \mathrm{C}_{6} \mathrm{H}_{5}+\mathrm{CO}_{3}(\mathrm{Mg} \mathrm{Hal})$.

Wie erwähnt, wirl die Cmlagering der Carbaminate sehr beeinflubt von Wasser. Wir verfuhren daher zunächst so, dath wir das Carbaminat in Bombenrühren einschlossen, um währed des Erhitzens jegliche Wasseranziehung zu vermeiden. Jas Einfuillen des meistens sehr zïhflüssigen und hygroskopischen Materials in die Röhren ist jeduch selur schwierig und verlustreich, wesha:lb wir nach einem anieren Verfahren suchten.

W'ir fanden, daß sich eine Umlagerung anch im offenen Kolhen erzielen läßst, wenn genügend lange und hoch erhityt und während der ganzen Daner der Reaktion trockne Kohlensäure eingeleitet wird. Die Ansheuten waren aber bei diesem Verfahren in allen Fällen recht mäßige. lies war vielleicht zum Teil dem Umstand zuzuscbreiben, dak der stetige Kohlensäurestron nicht so vä.lig getrocknet werlen liounte, dak or an die stark hygroskopischen Magnesiumsalze kein Wasser mehr abgegeben hätte, welches, wie oben erörtert, in Verein mit Kohlensïure außjerordentlich schädlich einwirlien mußte. Doch liamen wir noch anf eine andere Vermutung:

Weit schwerer als die Jodmagnesiunsalze lasset sich die Brontund noch schlechter die Chlorverbindungen unlagern. Anscheinend geht also die Umlagerung um so leichter voustatten, je schwerer die den Carboxylwasserstoff vertretende Gruppe ist. Manches läßt sich weiterhin dafïr ins Feld fübren, dal $B$ wenigstens eine der in den $A l-$ kymagnesiumsalzen komplex gebundenen Ïthermolekeln noch den dimit bereiteten Carbaminaten eigentünlich ist, wie etwa die folgende Formel auscirickt:

$$
\mathrm{C}_{6} \mathrm{H}_{5} \text {. CH. COO Mg. Hal, O }\left(\mathrm{C}_{2} \mathrm{H}_{5}\right)_{2} \text {. }
$$

Der Frsatz der Äthermolekel durch eine schwere Gruppe mübte dann von Vorteil für die Umlagerung sein köanen.

Diese lirwägung - die übrigens in keiner Weise mehr als eire Annahme sein will - fuihrte uns daza, den Ïther zunächst durcb Safrol, dann durch Chinolin zu ersetzeu. Li zeigte sich indessen, daß bei der hohen 'lemperatur, die zur Anwendung kam, diese Sub- 
stanzen selbst in Mitleidenscbaft gezogen wurden'). Wir mußten also, anders verfahren.

Tschelinzeff ${ }^{2}$ ) hat vor einiger Zeit eine Methode der Darstellung von Alkylmagnesiumbaloiden beschrieben, die sich mit Hülfe von tertiüren Aminen ohne $\mathrm{A}$ ther vollzieht. Leider hat das Verfahreu eine Reibe ron C̈belständen. Jedes Jodalkyl erfordert nach Tschelinzeff nämlich sein bestimmtes Amin. Überdies ist die Reaktiou fast immer nur partiell und benötigt trotzdem C̈berschuß an Alkyljodid. Sie wird in vielen Fällen außerdem noch dadurch beeinträchtigt, daß das Amin mit dem Jodid zu einem quaternären Sal\% zusammentritt, und schließlich sind nur die teuren Jodalkyle verwendbar.

Ls war daher von außerordentlicher Wijchtigkeit, als es sich ergab, daß alle diese C̈belstände fortfallen, wenn man das tertiüre Arylamin durch ein seliundäres ersetzt, nämlich dasjenige, welches man in Amidosäure ïberführen will, und sodann unter Erhitzen trockne Koblensïure einleitet. Das Magnesium verschwindet z. B. bei Anwendung von Metbylanilin im Verlauf mebrerer Stunden bis auf die letzte Spur. Man beoötigt nur die äquimolekulare Menge Jodalkyl, welches sich manchmal durch Brom- und in gewissen Fällen anscheinend auch durch Cbloralkyl ersetzen läßt. Etwa entstebende quaternäre Salze werden im Verlauf der Reaktion wieder zersetzt. Nan hat es z. B. beim Methyl-anilin mit folgenden Vorgängen zu tun: $\mathrm{C}_{6} \mathrm{H}_{3} \cdot \mathrm{NH} . \mathrm{CH}_{3}+\mathrm{CO}_{2}+\mathrm{Mg}+\mathrm{CH}_{3} \mathrm{~J}=\mathrm{C}_{6} \mathrm{H}_{5} \cdot \mathrm{N}(\mathrm{COOMgJ}) \cdot \mathrm{CH}_{3}+\mathrm{CH}_{4}$;

$$
\mathrm{C}_{6} \mathrm{H}_{5} . \mathrm{N}(\mathrm{COOMgJ}) \cdot \mathrm{CH}_{3} \quad \text { twa } 200^{\circ} \succ \mathrm{C}_{6} \mathrm{H}_{4}<\mathrm{COOMgJ} .
$$

(Durch das nötig werdende Eirhitzen tritt zugleich schon Umlagerung ein.)

Wie weitere Versuche zeigten, läßt sich das sekundäre Arylamin unter Umständen auch durch ein äquimolekulares Gemisch von primärem und tertiärem Arylamin ersetzen, so z. B. das Methylanilin durch ein Gemisch ron Anilin und Dimethyl-anilin, das sich verhält wie Monometbylanilin. Nur darf, falls die Synthese einer sekundären Aminosïure beabsicbtigt ist, kein Überschuß des Gemisches

1) Bezinglich des Chinolin: vergleiche man die Angaben von F. und L. Sachs, dieve Berichte 37, 3088 [1904], owie von B. Odda, Chem. Zentralhl. 1904, II, 836. Au Safrol "nt stehen interessante Verbindungen, über die demnächst berichtet werden soll.

$\Rightarrow$ Diw. Beriehte $37,4534[1904]$. Vergl. Tschelinzeff, liese Berichte 37, $2081[1904] ; 38,579[1905]: 39,773$ [1906]: 40, 1487 [19177]; 41, 646 [1:3:8]. 
oder von Dimethylanilin vorbanden sein. Die tertiäre Base wirlst sonst methylierend und verwandelt die sekundäre Amidosäure in tertiäre. So bat naan ein Mittel in der Hand, auch tertiäre Aminosäure zu syntbetisieren. Das Dimethylanilin wandelt sich dabei in Monomethylanilin, das sich gewinnen und in einer folgenden Operation aluf sekundäre Aminosäure verarbeiten läßt. Man vermag also die Synthese so zu gestalten, daß sie ohne Beeinträchtigung des Hauptprodukts ein für das gleiche Verfahren brauchbares Nebenprodukt liefert.

Aber auch die Anweudung des Halogenalkyls läßt sich, wie ans dem Folgenden ersichtlich, röllig vermeiden. Wie oben schon gesagt, reagiert z. B. das aus Methylanilin und Jodmethyl entstehende Jodmethylat beim Errbitzen mit Maguesium und Kohlensäure zun Teil so, als wenn es noch aus seinen Komponenten, Jodmethyl und Methylanilin, bestände. Nun ist aber das Jodmetliylat des Methylanilins identisch mit dem Jodhydrat des Dimethylanilins, kann also durch dieses ersetzt werden, und in der Tat ergibt sich in Wirklichkeit auch kein Unterschied in der Evtstehung von Aminosäure, ob man das eine oder das andere anwendet. Zugleich ist damit die unbequeme Handbabung von Chlor- und Bromalkyl umgangen. Denn statt z. B. ein sekundäres Arylamin und Chloräthyl anzuwenden, nimmt man das Chlorhydrat des tertïren Amins, was sich um so mehr empfieblt, als die tertiären Amine gewölnnlich weit zugänglicher sind als die sekuudären.

Was den in der zitierten vorläufigen Mitteilung von Houben angegebenen Versuch betrifft, so bildete Methylanilin das Ausgangsmaterial. Es wurde nittels einer ätherischen Lösung von Jodmethylmagnesium in seine Jodmagnesiumverbindung und diese durch Kohlensüure ins Carbaminat verwandelt. Dieses, durc's Erbitzen in Bombenrohr umgelagert, ergnb eine Aminosäure, die sehr der $p$-Dimethylaminobenzoesäure ähnelte. Liese konnte leicht aus primär gebildeter $\boldsymbol{p}$-Monometbylaminobenzoesäure eutstanden sein, da das angewandte überschüssige Jodmethyl metbylierend wirken mußte. Indessen stimmten die Analysenzablen - freilich ungenau, so daß seinerzeit von ibrer Veröffentlichung abgesehen wurde - besser auf die Nonomethylaminosüure. Und ror allem lieferte sie, was damals ausschlaggebend für die Auffassung der Säure erscheinen mulite, ein Nitrosamin. Dieser Unstand schien mit der Dimethylaminosäure unverträglich, denn E. Bischoff') hatte angegeben, da 3 die $p$-Dimethylaminobenzoesäure bei der Nitrosierung im Kern nitrosiert werde.

Indessen beschrieb kurze Zeit nach Erscheinen der vorläufigen Mitteilung von Houbeu M. Jaffép eine Säure als $p$-Metbylamino-

1) Dicse Berichte 22, 342 [1889].

2) Diese Berichte 38, 1208 [1905]. 
benzoesäure, die, auf physiologischem Wege gewonnen, ganz andere Eigenschatten zeigte als die von Houben aus Methylanilin gewonuene. Eigentümlicherweise stimmte aber der von Jaffé für das Nitrosamin seiner Säure angegebene Schmelzpunkt wieder ziemlich mit dem des Nitrosamins der rou Ilouben gewonuenen Säure überein, obgleich dieser Wert als unzuverlässig angeseben und daher nicht mitgeteift worden war.

Zur Aufklärung der Widersprïche wurden Versuche fortgesetzt, die schon gleich bei der Darstellung der Säure aus Methylanilin nebenher legonnen worden waren und bezweckten, die p-Methylaminobenzoesäure aus $p$-Aminobenzoesäure und Methylsulfat zu gewinnen. Wirklich reine sekundäre Säure auf diesem Wege zu erlangen, erwies sich als recht umständlich, und es wurden viele Versuche nötig. Wir waren nuch nicht ganz damit zu Ende gekommen, als eine Arbeit von Baudisch ) willkommene Aufklärung brachte und unter anderem die Unrichtigkeit der Angabe Bischoffs erwies, wonach die $p$-Dimethylaminobenzoesäure bei der Nitrosierung ein Kernnitrosoderivat liefere. Baudisch zeigte vielmehr, daß die Nitrosierung, wie wir inzwischen auch bereits gefunden batten, ziemlich kompliziert verläuft, und neben anderen Verbindungen auch das Nitrosamin der Monomethylaminobenzoesäure erzeugt. Äbnliche Beobachtungen, wonach bei der Nitrosierung Methylgruppen von Stickstoff abgespalten werden können, sind auch schon von anderer Seite, so von O. Fischer und E. Diepolder"), gemacht worden. Diese Autoren verweisen zugleich auf ältere Angaben dieser $\mathrm{Art}^{3}$ ).

Unsere Beobachtungen stimmen mit den Angaben Baudischs vollkommen überein. Danach ist die in der vorläufigen Mitteilung beschriebene, aus Methylanilin gewonnene Säure in wesentlichen $p$-Dimethylaminobenzoesäure, und die bei der Nitrosierung gewonnene $\boldsymbol{p}$-Methylnitrosaminbenzoesäure hatte sich demnach zum größten Teil durch Abspaltung eines Methyls vom Stickstoff gebildet.

Experimentelles.

Versuche mit Methyl-anilin.

I. Versuch der Isolierung von methyl-phenyl-carbaminsaurem Magnesiumjodür.

Nachdem es E. Mohr $)$ gelungen war, das phenylcarbaminsaure Barium zu isolieren, schien es möglich, auch andere Phenylcarbami-

1) Diese Berichte 39, 4293 [1906]. 2) Ann. d. Chem. 286, 163.

3) Jahresber. 1866, 415; diese Berichte 12, 1811 [1879]; 20, 2459 [1887].

†) Journ. f. prakt. Chem. [2] 72, 297; 73, 177 und 207. 
nate rein darzustellen. $Z u$ diesem $Z$ weck wurde in eine ätherische Methylmagnesiumjodidlïsung die entsprechende Menge Metbylanilin getropft, trocknes Koblendioxyd eingeleitet und das hierbei ausfallende bordeauxrote (̈), welches ein komplexes Magnesiumsalz der Methylphenylcarbaminsäure sein mußte, in absolutem Alkobol gelöst. Wasserfreier İther fïllte daraus einen weißen, pulvrigen Niederschlag, der schnell abgesangt, mit Äther gewaschen und im Exsiccator getrocknet wurde. Er zeigte sich löslich in Wasser, Aceton und Alkohol, nicht aber in Äther, Petroläther, Benzol und Chloroform. An der Luft färbte er sich rasch gelb, und er war so zersetzlich, daß eine stimmende Analyse nicht erbalten werden konnte. Doch srhien inmerhin der Haurtsache nach ein Carbaminat vorzuliegen.

$0.1314 \mathrm{~g}$ sbst.: $0.1215 \mathrm{~g} \mathrm{AgJ}$.

$\mathrm{C}_{8} \mathrm{H}_{8} \mathrm{O}_{2} \mathrm{NMg}$ J. Ber. J 42.14. Gef. J 49.96 .

Die wäßrige Lösung der Substanz gab mit einer wäßrigen Lösung von Chlorbarium einen weißen, Eisenchlorid einen rotbraunen, Kupferacetat einen graugrünen, Silbernitrat einen gelben Niederschlag (AgJ $:$ ).

Da die Ester der Carbaminsäuren die Salze derselben an Beständigkeit weit äbertreffen, wurde der Versuch gemacht, mittels Methylsulfats das Carbaminat in ein Urethan zu verwandeln, doch ohne Erfolg.

II. Umlagerung des Methyl-pbenyl-carbaminats.

a) Umlagerung im Bombenrohr. $18.2 \mathrm{~g}$ Magnesiumband und $106.2 \mathrm{~g}$ Methyljodid (etwas mehr als die berechnete Menge) wurden in $390 \mathrm{ccm}$ absoluten Äthers zur Reaktion gebracht. Nach Aufzehrung des Metalls wurde bei Fiskühlung und andauerndem Schütteln die berechnete Menge Methylanilin $(80 \mathrm{~g})$ zugetropft. Das Yethylanilinmagnesiumjodid fiel dabei als weiße Masse aus, wäbrevd reichlich ein brennbares Gas entwich (Methan). Scbließlich wurde durch gelindes Erwärmen auf dem Wasserbade die Reaktion zu Ende gefiihrt, sodann bei Wasser- oder Eiskiihlung scharf getrocknetes Kohlendioxyd eingeleitet, das gleichmäßig absorbiert wurde, während sich wie beim vorhergehenden Versuch ein bordeauxrotes Öl absehied. Dieses Öl wurde nach Abgießen des überstehenden Äthers in zugeschmolzenen Röhren 18-24 Stunden lang auf $180-220^{\circ}$ erbitzt. Der Inhalt bildete dann eine braune, homogene, schwach durchscheineude, gummöse Masse, die in verdüunter Essigsäure gelöst wurde. Dabei trat eine stürmische Gasentwicklung ein, die wabrscheinlich von der Zersetzung noch unveränderten Methylphenylcarbaminats herrührte. Die saure Lösung wurde ansgeäthert, wobei der Ätber eine starke 
blaue Fluorescenz annahm. Hierauf wurde der ätherische Auszug mit verdünnter Sodalösung extrahiert und die alkalische Lösung dann vorsichtig mit verdünnter Essigsäure angesäuert. Es fiel ein fast weißer, flockiger Niederschlag aus, der, abgesaugt, mit Wasser gewaschen und im Vakuum über Schwefelsäure getrocknet, $3.5 \mathrm{~g}$ wog. Da bei dem Umfüllen des zäbflüssigen, dazu gegen Feuchtigkeit und Luft sehr empfindlichen Carbaminats in die Bombenröbren stets ein beträchtlicher Teil zersetzt wird oder verioren geht, wird die Ausbeute stark beeinträchtigt.

Durch Umkrystallisiereu der ausgefällten Aminosäure aus schwach verdüntem Alkobol wurden weiße, etwas rötliche Nadeln vom Schmp. 229-2310 erhalten. Diese Säure mußte offenbar identisch sein mit derjenigen, für die Houben in seiner vorläufigen Mitteilung den Wert 228-229 angibt. Da wir etwas größere Mengen zur Verfügung hatten, gelang es, die Verbindung in größerer Reinheit darzustellen. Nach mehrmaliger Krystallisation aus Alkobol stieg der Schmelzpunkt bis auf $238^{\circ}{ }^{1}$ ). Die Analysen stimmten mit aller Schärfe auf eine $p$-Dimethylamino-benzoesäure.

0.1672 g Sbst.: $0.3998 \mathrm{~g} \mathrm{CO}_{2}, 0.1009 \mathrm{~g} \mathrm{H}_{2} \mathrm{O} .-0.1422 \mathrm{~g}$ Sbst.: $10.8 \mathrm{ccm}$ $\mathrm{N}\left(12 . \overline{5}^{0}, 715 \mathrm{~mm}\right)$.

$$
\begin{aligned}
& \mathrm{C}_{9} \mathrm{H}_{11} \mathrm{O}_{2} \mathrm{~N} \text {. Ber. C 65.41, H 6.71, N } 850 . \\
& \text { Gef. D } 65.30 \text {, "6.75, " } 8.50 .
\end{aligned}
$$

Die Eutstehung der Säure ist wohl so aufzufassen, daß sich aus den methylphenylcarbaminsauren Magnesiumjodür beim Erhitzen zunächst ein Salz der $p$-Yonomethylaminobenzoesäure bildet, die dann unter der Einwirkung eines methylierenden Agens in die Dimethylaminusïure übergeht. Als Metbylierungsmittel kommt vielleicht überschiissiges Jodmethyl in Betracht, desseu Anwesenheit vielleicht daraus zu erklären ist, daß die Reaktion zwischen Magnesium und Jodmethyl uicht ganz vollständig verlaufen war; docb ist nicht ausgeschlossen, daß aluch das methylphenylcarbaminsaure Magnesiumjodür methylierend auf $p$ - \ethylaminobenzuat wirkt.

b) Umlagerung des Methyl-phenyl-carbaminats in Dimethylanilin-Lösung und im Kohlensäurestrom. Die Verluste, die mit dem eben beschriebenen Veriahren verbunden sind, veranlaßten uns, die Umlagerung des Carbaminats durch Erhitzen desselben im Koblensäurestrom zu versuchen, zu welchem $Z$ wecke es in einem passenden Lösungsmittel gelöst werden muß. Wir verwandten hierzu sowohl Satrol, wie Chinolin und Dimethylanilin. Die beiden ersten erwiesen

1) I. Michler, diese Berichte 9, 401 [1876], gibt den Schmp. $235^{\circ}$ an. J. Pinnow, diese Berichte 32, 1408 [1899], findet 238-2390. J. Johnston, Chem. Zentralbl. 1905, II, 44, beobaclitet $235-236^{\circ}$. 
sich als unbrauchbar, da sie bei der zur Anwendung kommenden bohen Temperatur selbst angegriffen werden. Beim Safrol wird das Methylen der Methylendioxygruppe von einem der beiden Sauerstoffatome getrennt und teils mit dem Alkyl des angewandten Alkylmagnesiumhaloids, teils mit dem Halogenmagnesiumrest verbunden analog den Erfabrungen, die Grignard ${ }^{1}$ ) beim Äthylenoxyd gemacht hat. Chinolin wird in ein dunkelrotes Harz verwandelt. Brauchbar erwies sich das Dimethylanilin.

$11.5 \mathrm{~g}$ Magnesiumspäne, $200 \mathrm{ccm}$ Dimethylanilin, $50 \mathrm{~g}$ Methylanilin und $144 \mathrm{~g}$ Jodäthyl wurden zusammengegeben und unter Kühlung trockne Kohlensäure eingeleitet, die auffallend rasch absorbiert wurde. Sodann wurde im Kohlensäurestrom 15 Stunden lang auf $190-200^{\circ}$ erhitzt. Bei $100^{\circ}$ trat starke Gasentwicklung auf. Zugleich sublimierte in den Hals des Rundkolbens, in dem die Reaktion vor sich ging, und den aufgesetzten Kübler eine weiße Substanz in ziemlicher Menge. Nach dem Erkalten stellte der Kolbeninhalt eine durchscheinende braune, kolophoniumartige Masse vor, die kein metallisches Magnesium mehr enthielt. Die Zersetzung mittels verdünnter Essigsäure lieferte $28 \mathrm{~g}$ fast reiner weißer Amidosäure. Sie wurde einige Male ans Alkohol umkrystallisiert uud erwies sich als $p$-Dimethylamino-benzoesäure. Ihr Schmelzpunkt lag bei $232^{\circ}$. Die Analyse lieferte folgende Werte:

$0.1240 \mathrm{~g}$ Sbst.: $0.2985 \mathrm{~g} \mathrm{CO}_{3}, 0.0720 \mathrm{~g} \mathrm{H}_{2} \mathrm{O}$.

$$
\begin{array}{r}
\mathrm{C}_{9} \mathrm{H}_{11} \mathrm{O}_{2} \mathrm{~N} \text {. Ber. C 65.41, H } 6.71 . \\
\text { Gef. " 65.74, 》 } 6.49 .
\end{array}
$$

Berechnet man die Ausbeute auf das angewandte Magnesium, so erreicht sie $40 \%$ der theoretischen.

Das weiße Sublimat, welches sich im Kolbenhalse festgesetzt hatte, wog $12 \mathrm{~g}$ und war nach seinem Jodgehalt und Verhalten $\mathrm{zu}$ urteilen :

$$
\text { Phenyl-äthyl-methyl-ammoniumjodid. }
$$

Es wurle mehrfach aus Alkohol umkrystallisiert und bilkete dann glänzende weiße Blättehen, die sullimierbar und in Wasser lïslich waren.

0.3633 g Sbst.: $0.3355 \div$ Ag J.

$\mathrm{C}_{9} \mathrm{H}_{14}$ N.T. Ber. J 48.29. Gef. J 48.32.

Wie aus dem Mitgeteilten zu erseben, war bei weitem mehr Jodäthyl zur Anwendung gekommen, als dem Magnesium und dem Methylanilin entspracb. Es ergab sich indessen, da $\beta$ man die Mengenverhältnisse unbeschadet des Resultats so wählen kann, daß auf ein Atom Magnesium eine Molekel Jodalkyl und eine Molekel Metbyl-

1) Chem. Zentralbl. 1903, II, 105. 
anilin kommt. An Dimetbylanilin setzt man zweckmäßig das $\mathrm{Zwei-}$ bis Dreifache der angewandten Menge Methylanilin zu. Leitet man nun unter Erwärmen auf $190-200^{\circ}$ (Ölbad) trockne Koblensäure zu, so wird im Verlaufe weniger Stunden das Metall völlig aufgezehrt, und die Bildung der Amidosäure geht langsam vonstatten.

c) Verfabren unter Anwendung von Methyl-anilin ohne Dimethylanilin. Äquivalente Mengen Magnesiumspäne und Jodmethyl werden mit dem $\mathrm{Zweifachen} \mathrm{der} \mathrm{äquivalenten} \mathrm{Menge} \mathrm{Mono-}$ methylanilin im Koblensäurestrom 15 Stuoden auf $190-200^{\circ}$ im ÖLbad erhitzt. Nach dem Erkalten wird der entstandene Kuchen durch Erhitzen mit Salmiaklösung zersetzt und die sowohl das entstandene Amidobenzoat wie die Magnesia in Lösung haltende Flüssigkeit mit verdünnter Essigsäure gefällt. So erhält man die substituierte $\boldsymbol{p}$-Aminobenzoesäure in etwa 43-prozentiger Ausbeute.

Während aber bei dem Verfahren, nit Dimethylanilin nur die Dimethylamino-benzoesäure nachgewiesen werden konnte, war hier in erheblicher Menge, ja anscheinend der Hauptsache nach, $p$-Monomethylamino-benzoesäure entstanden, denn bei der Nitrosierung ging das Produkt fast quantitativ in $p$-Methylnitrosaminbenzoesäure über. Zwar vermag, wie schon erwähnt, auch die $p$-Dimethylaminobenzoesäure bei der Nitrosierung in die genannte Nitrosaminsāure überzugehen. Doch bildet sich dieselbe durchaus nicht quantitativ, meistens sogar nicht über $30 \%$.

Um über die Methylaminobenzoesäure Klarheit zu gewinnen, studierten wir zunächst die Methylierung der $p$-Amidobenzoesäure mit Hilfe von Metbylsulfat.

Methylierung der $p$-Amido-benzoesäure mit Methylsulfat in wä $B$ rig-alkalischer Lösung.

$10 \mathrm{~g}$ reine $p$-Amidobenzoesäure, $50 \mathrm{ccm}$ Wasser, $3 \mathrm{~g}$ Natronbydrat wurden zusammengegeben und nach Bildung. einer klaren Lösung im verschlossenen Rundkolben mit der berechncten Menge Methylsalfat, $9.2 \mathrm{~g}$, kräftig geschüttelt, cine halbe Stunde auf dem Wasserbade an Steigrohr erwärmt und 24 Stunden stehen gelassen. Wenige Minuten nach Zugabe des Methylsulfats begann sich die Reaktion bereits durch Ausscheidung eines weißen Niederschlags anzuzeigen. Der Niederschlag wurde abgesaugt, mit Wasser gewaschen und im Vakunmexsiccator über Schwefelsäure getrocknet. Fr wog sodann $9 g$, begann schou bei $140^{\circ} \mathrm{zu}$ sintern und schmolz teilweise hei $147-150^{\circ}$, der letzte Teil aber erst bedeutend höher, bei $195^{\circ}$. Es lag also offenbar ein Gemisch vor, aus welchem sich in der Tat $1.8 \mathrm{~g}$ Dimethylaminobenzoexäure isolieren ließen. $\mathrm{Zu}$ diesem Zwecke wurde die Substanz mit $500 \mathrm{ccm}$ Wasser gekocht, wodurch die $\boldsymbol{p}$-Meth ylamin osä ure in Lösung geht, während fast alle Dimethylaminosäure zurückbleibt. Diese wurde aus Alkohol krystallisiırt und zeigte dann den Schmp. 238 . Aus der alkoholischen Mutterlauge lich 
sich mit Wasser noch $4.5 \mathrm{~g}$ Sbst. fällen, die mit Hille verdünnter Schwefelsäure in dem eben genannten wäßrigen Auszug gelöst wurdea. Diese Lösunğ mußte sämtliche entstandene Monomethylaminobenzocsäure enthalten. In der Meinung, sic als Nitrosaminsäure abscheiden und von allen Beimengungen tremen zu können, versetzten wir die saure Lösung unter Eiskühlung mit einer eiskalten Lösung von $5 \mathrm{~g}$ Natriumnitrit. Es fiel in Mengen eine relbe Substanz aus, die nach viermaligen Uumkrystallisieren aus Alkohol schöne, glänzende, braune Nadeln rom Schmp. $206^{\circ}$ hildete. Obschon der Schmelzpunkt $10^{0}$ höher liegt, als Jafl'́l) jhn für clie $p$-Methylnitrosaminbenzoesäure anquibt, zeigte die substanz im ülıigen ähnliche Eigenschaften, krystallisierte aus viel Wasser in kleinen gelben Närlelchen und gab bei der Analyse stimmende Zahlen.

$0.1210 \mathrm{~g}$ Sbst.: $0.2373 \mathrm{~g} \mathrm{CO}_{2}, 0.0503 \mathrm{~g} \mathrm{H}_{2} \mathrm{O}$ - $-0.1430 \mathrm{~g}$ Sbst : $0.2797 \mathrm{~g}$ $\mathrm{CO}_{2}, 0.0615 \mathrm{~g} \mathrm{H}_{2} \mathrm{O} .-0.1765 \mathrm{~g}$ Sbst.: $23.2 \mathrm{~cm} \mathrm{~N}\left(15^{\circ}, 761 \mathrm{~mm}\right) .-0.2207 \mathrm{~g}$ Slbst.: $30.9 \mathrm{ccm} \mathrm{N}\left(15^{\circ}, 724 \mathrm{~mm}\right)$.

$$
\begin{array}{llll}
\mathrm{C}_{8} \mathrm{H}_{8} \mathrm{~N}_{2} \mathrm{O}_{3} . & \begin{array}{l}
\text { Ber. C } 53.29, \\
\text { Gef. " } 53.56,53.42,
\end{array} & \mathrm{H} 4.48, & \mathrm{~N} 15.55 . \\
&
\end{array}
$$

Obgleich aber der Schmelzpunkt konstant war und die Analysen stimmter, war die Substanz nicht im mindesten einheitlich, was vor dem Erscheinen der Arbeit von Baudisch festzustellen, außerordentlich viel Mühe machte. Der ron uns eingeschlagene Weg zur Reindarstellung der Nitrosaminsäure ist unten angegeben.

\section{Methylierung in eisessigsaurer Lösung.}

Houben und Brassert ${ }^{2}$ ) baben ein Verfahren zur Methylierung doy Antimanilsäure in Eisessig angegeben. Wir versuchten auf ähnliche Weise auch die $p$-Amido-benzosäure in ibr Monomethylderivat zu verwandelı. Während aber bei der Methylierung der Anthranilsäure nach dem erwähnten Verfahren im wesentlichen nur Monomethylanthranilsäare erhalten wird, entstehon boi der gleichen Behandlung der $p$-Amidobenzoesiaure Gemische, die primäre, sekundäre und tertiäre Säure enthalten.

$18 \mathrm{~g} p$-Amidobenzoesäure wurden in $100 \mathrm{ccm}$ Fisessig gelöst, abgekühlt und mit der berechneten Menge Methylsulfat, $16.6 \mathrm{~g}$, versetzt, darauf 30 Minuten lang am Rückflußkühler gekocht, wobei sich reichlich ein weißes Salz ausschied, dessen Menge sich betm Erkalten und 24-stündigen Stehen noch bedeutend vermehrte. Der Niederschlag wurde nach dieser Zeit abgesaugt, mit Fisessig ausgewaschen und mit ahsolutem Äther nachgespült. Getrocknet wog er rund $10 \mathrm{~g}$. Fr erwies sich als das

$$
\text { Sulfat der } p \text {-Amidobenzoesãure, } \mathrm{SO}_{4} \mathrm{H}_{3}\left[\mathrm{C}_{6} \mathrm{H}_{4}\left(\mathrm{NH}_{2}\right) \cdot \mathrm{COOH}\right]_{2} \text {, }
$$

nicht aber etwa als ein methylschwefelsaures Salz. Trotz rer Abwesenheit von Wasser war also auch das zweite Methyl des Methylsulfats abgespalten worien. Daher lag die Annahme nahe, es werde sich viel Dimethylamino-

) loc. cit. 2) Diese Berichte 39, 3234 [1906]. 
henzoenäure gedildet haben. Doch wies ein starkel Geruch nach Methylacetat nuch auf einen anderen Verbleib des Methyls hin.

Da: iulfat wurde zweimal aus 50 prozentigem Alkohol krystallisiert und so in schönen, schnerweißen Blättchen (unter lem Mikroskup hexagonale Plittchen) erhalten. Es läßt sich auch gut aus Wasser krystallisieren, ist aher clann ledeutend weniger halthar und wird schon in kurzer Zeit golb und haun. Beim Erhitzen zersetzt es sieh, ohne zu schmelzen. $\mathrm{SO}_{+} \mathrm{Ba}$

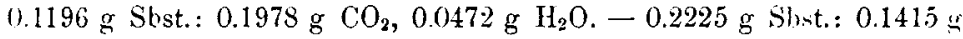

$$
\begin{aligned}
& \mathrm{C}_{14} \mathrm{H}_{16} \mathrm{~N}_{2} \mathrm{O}_{8} \rightarrow \text {. Ber C 45.13, H 4.33, S 8.62. } \\
& \text { Gef. „45.17, 》4.41, \$ } 8.74 \text {. }
\end{aligned}
$$

Ls sei nicht unerwähnt gelassen, daß schon einige Beobachtungen vorliegen, wonach metbylscbwefelsaure Salze organischer Basen beim Erhitzen sich in Sulfate verwandeln. Ähnliches fand Gräbe ${ }^{1}$ ) bei Phenolen.

$$
p \text { - Metbylnitrosamin-benzoesäure, } \mathrm{CH}_{3} \cdot \mathrm{N}-\longrightarrow-\mathrm{COOH} \text {. }
$$

Ia $10 \mathrm{~g}$ des eben beschriebenen Sulfats $7.4 \mathrm{~g}$ Amidosäure eutsprechen, konnten höchstens $10.6 \mathrm{~g}$ Amidosäure methyliert worden sein. Jie Methylierungsprodukte mußten sich in der essigsauren Mutterlauge befinden. Das Filtra: wurde daher mit Eis gekiult und sodann nit einer eiskalten Iösung von $6 \mathrm{~g}$ Natriumnitrit in $20 \mathrm{ccm}$ Wasser rersetzt, 10 Minuten in Eis stehen gelassen und hierauf in eiv Liter eiskaltes Wasser gegossen. Der ausfallende gelbe Niederschlag wurde nach 10 Minuten abgesaugt, mit Wasser gewascben und aus $150 \mathrm{ccm}$ siedendem 96-prozentigem Weingeist umkrystallisiert. Beim Abkühleu schieden sich 7.5 s der, wie oben erwähnt, nicht einheitlichen Nitrosaminsüure aus, während noch $1.5 \mathrm{~g}$ unreinerer Substanz. durch Ahdunsten des Filtrats auf dem Wasserbade erhalten wurden. Die reinere Substanz machte wiader den Eindruck eines einheitlichen Körpers, zeigte den Schnp. 202-203, der sich beim Umkrystallieren nur unwesentlich änderte, und bestand aus glänzenden, brauneu Nadelu.

C̈bergießt man die S̈̈ure mit 10-prozentigem wäßrigem Ammoniak, so findet erst bein Erwämen eine Iösung statt. Die rotbraune Flüssigkeit scheidet beim Abkiihlen goldgelbe Blätchen aus, die erst bei Zugabe ziemlicher Wassermengen wieder in Lösung gehen, auf Zusatz von festem oder gelöstem Salmiak aber wieder in reicblicher Menge berausliommen. Das Salz wurde durch Abkühlung der ammoniakalischen Lösung obne Anwendung ron Salmiak abgeschieden,

9) Ann. d. Chem. 340, 207. 
abgesaugt, mit Alkohol und Äther gewaschen, einige Stunden an freier Luft belassen und alsdann analysiert.

$0.1171 \mathrm{~g}$ Sbst.: $0.2103 \mathrm{~g} \mathrm{CO}_{2}, 0.0620 \mathrm{~g} \mathrm{H}_{2} \mathrm{O} .-0.1181 \mathrm{~g}$ Sbst.: $22.2 \mathrm{l}$ $\mathrm{N}\left(14^{\circ}, 719 \mathrm{~mm}\right)$.

$$
\begin{array}{ll}
\mathrm{C}_{8} \mathrm{H}_{11} \mathrm{~N}_{3} \mathrm{O}_{3} . & \text { Ber. C } 48.6 \mathrm{~S}, \mathrm{H} 5.62, \mathrm{~N} 21.35 . \\
& \text { Gef. » } 49.05, \text { » } 5.92, \text { » } 1.01 .
\end{array}
$$

Das Salz verliert beim Erhitzen seine goldgelbe Farbe und wird unter Nebelbildung heller. Es schmilzt beim Erhitzen im Capillarrohr bei $215-217^{\prime}$ und scheint dabei in die freie Süure überzugeben, die sich weiterbin zersetzt. Unter dem Mikroskop zeigt das Salz langprismatische Nadeln.

Hat man das Ammoniumsalz mehrmals aus Ammoniaklösung unikrystallisiert, so löst es sich in Wasser fast völlig farblos auf, was darum auffallend erschien, weil die Nitrosaminsüure der oben beschriebenen Bereitungsart sich in verdünnter Ammoniaklösung tiefrot auflöst. Das sich farblos lösende Salz wurde daher in Wasser gelöst und mit verdünnter Lssigsäure die Nitrosamiusäure wieder gefällt. Sie zeigte sich in der Tat von der ursprünglich angewandten Nitrosaninsäure beträchtlich verschieden. Aus Alkobol krystallisierte sie

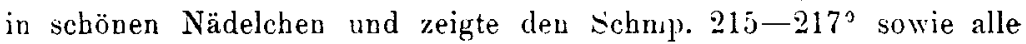
Figenschaften, die Baudisch für die reine $p$-Methylnitrosaminbenzoesäure angibt. Freilich waren diese Angaber damals noch nicht veröffentlicht.

Dieser Weg ist vielleicht der kürzeste und einfachste, um von der rohen zur reinen $p$-Methylnitrosamin-benzoesäure zu gelangen. Doch bedarf man einer nicht zu geringen Substanzmenge.

$0.1069 \mathrm{~g}$ Sbst.: $0.2076 \mathrm{~g} \mathrm{CO}_{2}, 0.0449 \mathrm{~g} \mathrm{H}_{2} \mathrm{O}$. $-0.0926 \mathrm{~g}$ Sbst. : $12.4 \mathrm{ccm}$ N $\left(21^{0}, 762.5 \mathrm{~mm}\right)$.

$$
\begin{array}{ll}
\mathrm{C}_{8} \mathrm{H}_{8} \mathrm{~N}_{2} \mathrm{O}_{3} . & \text { Ber. C 53.29, H 4.48, N } 15.58 . \\
& \text { Gef. » } 33.04, » 4.70, \text { » } 15.41 .
\end{array}
$$

Reduziert man die reine Nitrosaminsäure mit verdünnter Salzsäure und Zink oder Magnesium, so wird die Nitrosogruppe eliminiert, und man gelangt zur $p$-Methylamino-benzoesäure. Wir haben es indessen vorgezogen, einen Umweg einzuschlagen, der uns diese Säure nicht nur absolut rein lieferte, sondern auch eine bequeme Isolierung gestattete und uns schließlich einige gut charakterisierte Derivate in die Hand gab.

$$
\begin{gathered}
\text { p-Nethylamino-benzoesäure-äthylester-Chlorhydrat, } \\
\text { HCl, } \mathrm{CH}_{3} \text {. NH. } \mathrm{C}_{6} \mathrm{H}_{4} \text {. COOC } \mathrm{H}_{5} \text {. } \\
\text { (Nit Walter Brassert.) }
\end{gathered}
$$

$5 \mathrm{~g}$ der rohen Nitrosaminsäure rom Scbmp. 200--2050 wurdem meblfein zerrieben und in kleinen Fortiones in $150 \mathrm{ccm}$ nicht ganz 
mit Chlorwasserstoff gesättigten absoluten Alkuhols eingetragen. Die Lösung ging nur langsam ronstatten, dnch war sie nach zwei Tagen vollendet. Die Flüssigkeit hatte eine rotbraune Farbe angenommen, und in Kolben wurde wiederholt starker Gasdruck beobachtet. (Die Reaktionsflüssigkeit befand sich in einem verschlossenen Rundkolben.) Nach weiteren drei 'lagen began die Ausscheidung großer, farbloser, durcbsichtiger Tafeln, die sich während längerer Zeit noch langsam vermehrten. Nach zehn Tagen wurden dieselben abgesaugt und mit absolutem Äther gewaschen. Sie erwiesen sich ohne weitere Behandlung als fast reines $p$-Methylamino-benzoesäureäthylester-chlorhydrat. Die Nitrosogruppe war also glatt eliminiert, gleichzeitig das Carboxyl verestert worden.

$0.1901 \mathrm{~g}$ Sbst.: $0.3853 \mathrm{~g} \mathrm{CO}_{2}, 0.1070 \mathrm{~g} \mathrm{H}_{2} \mathrm{O}$. $-0.1579 \mathrm{~g}$ Sbst.: $9 \mathrm{~cm}$ $\mathrm{N}\left(18.5^{0}, 744.7 \mathrm{~mm}\right) .-0.1204 \mathrm{~g}$ Sbst.: $0.0806 \mathrm{~g} \mathrm{Ag} \mathrm{Cl}$.

$$
\mathrm{C}_{10} \mathrm{H}_{14} \mathrm{O}_{2} \mathrm{NCl} \text {. Ber. C 55.66, H 6.54, N 6.51, Cl } 16.44 \text {. }
$$

Gef. 》 55.28 , 》6.30, 》6.45, 》 16.55 .

Das Chlorhydrat schmolz scharf bei $173^{\circ}$. Es wog $1.5 \mathrm{~g}$ und war von rein weißer Farbe.

Geht man von annähernd reiner Nitrosaminsäure aus, so kann man das Chlorbydrat leicht in einer Ausbeute von etwa $80 \%$ der theoretischen erbalten. Die alkoholisch-salzsaure Jösung erfültt sich dann mit einem Krystallbrei des Salzes, welches so direkt rein gewonnen wird. Bei dem oben beschriebenen Versuch wurden außer den $1.5 \mathrm{~g}$ reiven noch $2.95 \mathrm{~g}$ fast reinen Chlorhydrats aus der alkoholisch-salzsauren Mutterlauge gewonnen, indem das Lösungsmittel abgedunstet und der dickflüssige Rückstand mit absolutem Äther getïllt wurde. Die Ausbeute betrug also im ganzen $4.45 \mathrm{~g}$, das sind $74 \%$ der aus $5 \mathrm{~g}$ Nitrosaminsäure zu erwartenden Höchstmenge. Zur Reinigung nicht ganz weißen Chlorhydrats erwiesen sich Aceton und Benzol als geeignete Krystallisationsmittel.

Aus dem Gewicht des gewonnenen Chlorhydrats darf man scbließen, da $\beta$ die rohe Nitrosaminsäure vom Schmp. 200-2050. so wie sie durch direkte Methylierung der $p$-Amidobenzoesäure mit Methylsulfat und Nitrosierung des entstehenden Gemisches ron Amidosäuren erhalten wurde, unindestens zu $74 \%$, reine Nitrosaminsäure enthalten hatte. Es muß indessen darauf aufmerksam gemacht werden, daß nan nicht immer zu solchen Resultaten gelangt, wie eine Reibe ron Versuchen gezeigt Lat. Manchmal entstebt nämlicb bei der Nitrosierung eine Substanz, die vielleicht $o$-Nitro-p-methylamino-benzoesäure ist. Diese Verbindung scheint sich unter der Einwirkung des alkoholischen Chlorwasserstoffs ebenfalls zu verestern und als Chlorhydrat dann zugleich mit dem beschriebenen Chlorhydrat ausfallen zu können. 
Jedenfalls erhält man unter Umständen ein Gemisch zweier Chlorhydrate, wovon das eine, vämlich das Nitroderivat, dadurch gekennzeichnet ist, daß es mit Wasser dissoziiert.

$$
\begin{gathered}
p \text { - Metbylnitrosamin-benzoesäure-äthylester, } \\
\qquad \mathrm{CH}_{3} . \underset{\mathrm{N}}{\mathrm{N}} \mathrm{C}_{6} \mathrm{H}_{4} \cdot \mathrm{COOC}_{2} \mathrm{H}_{5} . \\
\text { NO }
\end{gathered}
$$

$2.7 \mathrm{~g}$ des $p$-Methylamino-benzoesïureäthylester-chlorbydrats wurden in reichlich W'asser unter Zugabe verdünnter Salzsäure gelöst und mit einer wäßrigen Lösung von $0.9 \mathrm{~g}$ Natriumnitrit in der Tiskälte nitrosiert. Es fiel sngleich ein rein weißer Niederschlag aus, der abgesaugt und mit riel Wasser gewaschen, sodann im Exsiccator getrocknet wurde. Er wurde aus Benzol krystallisiert und ergab $1.7 \mathrm{~g} p$ Methylnitrosamin-benzoesäureäthylester, lange, zu Büscheln vereinigte Nadeln, fast farblos, vom Schmp. 55.5 . Zur vollständigen Reinigung wurde die Substanz in wenig absolutem Äther gelöst und die filtrierte Lösung stark eingeengt, sodann mit Petroläther gefällt, der ausfallende, fast weiße Niederschlag aus Petroläther krystallisiert und so in langen, fast farblosen Nadeln rom Schnp. 570 (Capillarrohr) erhalten. Das Newicbt der Verbindung betrug alsdann noch $1.1 \mathrm{~g}$.

$0.1073 \mathrm{~g}$ Sbst.: $0.2270 \mathrm{~g} \mathrm{CO}_{2}, 0.0572 \mathrm{~g} \mathrm{H}_{2} \mathrm{O} .-0.1151 \mathrm{~g}$ Sbst.: $14.6 \mathrm{ccm}$ N $\left(25^{\circ}, 719 \mathrm{~mm}\right)$.

$$
\begin{aligned}
& \mathrm{C}_{20} \mathrm{H}_{12} \mathrm{O}_{3} \mathrm{~N}_{2} \text {. Ber. C 57.65, H .5.81, N 13.4s. } \\
& \text { Gef. 》5 .78, 》5.96, * } 13.43 .
\end{aligned}
$$

$$
p \text {-Metbylamino-benzoesäure. }
$$

$1.1 \mathrm{~g}$ des aus Aceton umkrystallisierten $p$-Methylamino-benzoesäureäthylester-chlorhydrats rom Schmp. $152^{\circ}$ wurden mit $25 \mathrm{ccm}$ 15-prozentiger Natroulange 10 Minuten lang am Rückflnßkühler gelinde gekocht. Das Chlorbydrat verwandelte sich in Öltropfen und löste sich völiig auf. Die Flüssigkeit wurde sodann mit dem gleichen Volumen Wasser verdüunt, von Verunreinigungen alffiltriert und zu dem noch warmen Filtrat ïberschüssige verclünte Essigsäure gegeben. Erst beim Erkalten schied sich die $p$-Methylaminosäure weiß und amorph aus. Sie wurde nach dem Trocknen im Exsiccator in heifiem Beuzol gelöst und so von etwas anorganischer Beimengung getrenut. Beim Erkalten der Benzollösung krystallisierte die Säure in atlasgläuzenden, besenförmig verzweigten Nädelchen. Der Schmelzpunkt war $162^{\circ}$ (Capillarrohr); die Ausbeute betrug $0.4 \mathrm{~g}$. Um sicher zu sein, vollkommen reine $p$-Methylaminobenzoesäure in Händen zu haben, haben wir sie noch ein zweites Mal aus Benzol krystallisiert. 
0.1355 g Sbst.: $0.3149 \mathrm{~g} \mathrm{CO}_{2}, 0.0730 \mathrm{~g} \mathrm{H}_{2} \mathrm{O}$. $0.1253 \mathrm{~g}$ Sbst.: $10.1 \mathrm{~cm}$ $\mathrm{N}\left(22.5^{\circ}, 760.4 \mathrm{~mm}\right)$.

$\mathrm{C}_{8} \mathrm{H}_{9} \mathrm{O}_{2} \mathrm{~N}$. Ber. C 63.52, $\mathrm{H} 6.02, \mathrm{~N} 9.29$.

Gef. 》63.38, »6.04, »9.16.

Der Schmelzpunkt wurde voch einmal mit einem verkiurzten Auschützschen Normalthermometer genommen und zu $160^{\circ}$ (korr.) gefunden. Beim Schmelzen tritt Gasentwicklung, vorher, bei $159^{\circ}$, Iilafärbung auf. Der Erstarrungspunkt wurde zu $158^{\circ}$ gefunden (Capillarrohr).

In einer besonderen Operation wurden abermals ca. $3 \mathrm{~g}$ Metbylamino-benzoesäureäthylester-chlorhydrat auf die beschriebene Weise in Methylaminosälre verwandelt und so über $1 \mathrm{~g}$ der reinen Säure gewonnen. Aus Benzol krystallisierte sie in zollangen, feinev, glänzendeo Nadeln und zeigte den Schmp. $161^{\circ}$ (korr.).

Der von M. Jafféd angegebene Schmelzpunkt $155-157^{\circ}$, ist nur wenig niedriger. Bestinmt unrichtig ist dagegen die Angabe rou J. Johnston ${ }^{2}$ ), 144-145?.

Die Säure ist bezüglich ihres Schmelzpunktes außerordentlich empfindlich gegen die geringsten Verunreinigungeu. Präparate. die bei der Analyse gut stimmende Werte liefern, weisen oft erbelilich erniedrigte Schmelzpunkte auf.

$$
p \text { - Methylamino-benzoesäure-athylester. }
$$

Um den noch unbekannten. Ithylester der $p$-Methylaminobenzoesäure $\%$ gewinnen, versetzten wir $7 g$ des Chlorhydrats, wie es durch Einwirkung ron alkoholischer Salzsäure auf rohe $p$-Methylnitrosaminbenzoesäure entstanden war, mit wïBriger Natriumactatlösung. Dor ausgeschiedene, schwach gellyliche Niederschlas wurde mehrmals ans Petroläther krystallisiert und so in farblosen, lanzettförmigren, meist rosettenförmig aggregrierten Blättern und Spachteln vom Schmp. 65-670 (Capillare) erhalten.

$0.1111 \mathrm{~g}$ Sbst.: $0.2736 g \mathrm{CO}_{2}, 0.0730 \mathrm{~g} \mathrm{H}_{2} \mathrm{O} .-0.1936 \mathrm{~g}$ Sbst.: $14.1 \mathrm{ccm}$ $\mathrm{N}(220,720 \mathrm{~mm})$.

$$
\begin{aligned}
\mathrm{C}_{10} \mathrm{H}_{13} \mathrm{NO}_{2} . & \text { Ber. } \mathrm{C} 66.99, \mathrm{H} 7.31, \mathrm{~N} 7.84 . \\
& \text { Gef. } \$ 67.26,>7.35, \diamond \mathbf{7 . 8 7 .}
\end{aligned}
$$

Der Ester löst sich in den meisten organischen Lösungsmitteln.

Vergleich der durch Umlagerung aus Carbaminat entstandenen Aminosäure mit der aus $p$-Amino-benzoe-

$$
\text { säure gewonnenen. }
$$

(J. Houben und A. Schottmiller.)

Turch Nitrosierung der Aminosäure, die obne Anwendung von Dimethylanilin aus Monomethylanilin dargestellt worden war, wurden

1) Diese Berichte 38, 1208 [1905].

2) Proc. Chem. Soc. ㄴ1, 156; Chem. Zentralbl. 1905, II, 44; 1906, II, 1006. 
reichlich $23 \mathrm{~g}$ einer Roh-Nitrosaminsäure gewonnen, ron welcher ein Teil in der bereits beschriebenen Weise mit alkcholischem Chlorwasserstofi behandelt wurde. $8.5 \mathrm{~g}$ fein zerriebener Nitrosaminsäure wurden im Laufe mehrerer Tage in $200 \mathrm{~cm}$ absoluten, mit Chlorwasserstoff fast gesättigten Alkohols eingetragen. die nach 10 Tagen abgeschiedene Krystallmasse algesaugt und aus der Mutterlauge durch Zusatz von Petroläther noch eine weitere Menge gewonnen. Diese Krystallmasse bestand fast nur aus $p$-Metbylamino-benzoesäureäthylester-chlorhydrat. Doch war ein anderes Chlorhydrat beigemischt wahrscheinlich das des o-Nitrometbylamino-benzoesäureäthylesters weshalb die Analysen keine stimmenden Werte ergaben. Auch durch Unkrystallisieren aus Benzol gelangte man nicht zu einem analysenreinen Präparat. Das Rohprodukt wurde daher durch etwa einstündiges Kochen mit verdünnter Natronlauge verseift. Durch vorsichtiges Ansäueru kann man dann die $p$-Methylaminobenzoesäure ausfällen. Besser versetzt man zunächst mit einem Überschusse verdïnnter Salzsäure, so daß die ausfallende Methylaminosäure wieder in Lösung geht. Ein etwa bleibender Niederschlag wird abfiltriert. Nun versetzt man die salzsaure Lösung mit soviel Natriumacetatlösung, daß die Mineralsäure eben gebunden wird. Nach einiger Zeit erfült sich die Flïssigkeit mit langen, weißen Nadeln, die von der Oberfläche her leicht rosige Farbe annehmen, weshalb man sie vor Zutritt der Luft schütr.t. Die Säure wog, nach dem Absaugen und Auswaschen getrocknet, $4.4 \mathrm{~g}$ und zeigte den Schmp. $160-162^{\circ}$. Sie hatte dieselben Eigenschaften wie die aus $p$-Amidobenzoesäure durch Metbylierung dargestellte. Mit dieser gemischt, zeigte sie nicht die mindeste Schmelzpunktdepression.

Darstellung der $p$-Methylamino-benzoesäure aus einem $G$ emisch von Anilin und Dimethylanilin.

Für späterhin in Aussicht genommene Versuche mit anderen Amiven schien die Möglichkeit wichtig, ein sekundäres Amin durch ein Gemisch von primärem und tertiärem ersetzen zu können. Wir wandten daher in einem besonderen Versuche statt des Methylanilins ein Gemisch ron Anilin und Dimethylanilin an und konnten auch hierbei ein gutes Resultat erzielev.

$6.6 \mathrm{~g}$ Magnesiumspäne wurden mit Hilfe von $38.2 \mathrm{~g}$ Metbyljodid in $100 \mathrm{ccm}$ absoluten Äthers gelöst, dann $66 \mathrm{~g}$ Dimethylanilin und $25 \mathrm{~g}$ Anilin zugesetzt, die Temperatur unter Einleiten trockner Kohlensäure langsam mittels Ölbades gesteigert und scbließlich etwa 15 Stunden lang im Kohlensäurestrom auf $200-220^{\circ}$ erhitzt. Bei der Zersetzung ergaben sich $10 \mathrm{~g}$ Aminosäure, die bei der Nitrosierung 
$11.5 \mathrm{~g}$ rohe Nitrosaminsäure liefertes. Die Mutterlauge, aus der die Aminosäure ausgefällt worden war, wurde ebenfalls nitrosiert und so noch $3.5 \mathrm{~g}$ Nitrosaminsäure erbalten. Die Gesamtausbeute au unreiner Nitrosaminsäure rom Schmp. $204^{\circ}$ betrug also $15 \mathrm{~g}$. Es muß in diesem lalle unentschieden bleiven, ob die Nitrosaminsäure aus sekundïrer oder aus tertiärer Säure entstanden ist. Jedenfalls ist aus dem Resultat des Versucbs zu erseben, daß auch ein Gemisch von Anilin und Dimethylanilin zur Darstellung von methylierter $p$-Aminobenzoesäure dienen kann. Nach später gemachten Erfahruvgen muß die entstandene Metbylaminobenzoesïure jedenfalls auch hier eine beträchtliche Menge Dimethylaminobenzoesäure enthalten haben.

\section{Überführung von Dimethylanilin-jodhydrat in p-Dimethylamino-benzoesäure.}

Wie aus deu früberen Versuchen hervorgeht, muß bei der Einwirkung ron Jodmethyl auf Methylanilin mit der Entstebung ron Methylanilinjodmethylat gerechnet werden. Das Methylat ist offenbar befäbigt, unter dem Einflusse von Magnesium und Kohlensäure bei höherer Temperatur in eine Aminobenzoesäure überzugeben. Da es nun mit Dimethylanilinjodhydrat identisch ist, so muß auch dieses unter den genannten Bedingungen zu dieser Synthese geeignet sein.

Zur Bereitung von

Dimethylanilin-jodhydrat,

welches in der Literatur merkwirdigerweise noch nicht beschrieben ist, wurde Dimethylanilin mit einem geringen Überschusse konzentrierter Jodwasserstoffsäure allmäblich unter Kühlıng gemischt, die entstandene zäbflüssige Masse in der eben geuiggenden Menge Alkohol gelöst und die Lösung abgekühlt; das sich ausscheidende Salz wurde je einmal ans Alkohol und aus Aceton umkrystallisiert, abgesaugt und mit etwas İther gewaschen. So wurden ziemlich beträchtliche Mengen des Jodhydrats in schönen, weißen Blätchen vom Schmp. $150^{\circ}$ (Capillarrohr) grewonnen.

\section{$0.1796 \mathrm{~g}$ Sbst.: $0.1695 \mathrm{~g} \mathrm{Ag} \mathrm{J}$.}

$\mathrm{C}_{8} \mathrm{H}_{12} \mathrm{~N}$ J. Ber. \& 51.00 . Gef. J 50.99 .

$20.7 \mathrm{~g}$ dieses Salzes wurden mit $20 \mathrm{~g}$ Dimetbylanilin und $2 \mathrm{~g}$ Magnesiumspänen gemischt. Beim Erwärmen des Gemisches in einem Ruvdkolben mit Steigrohr und Chlorcalcium-Verscbluß trat Gaseutwicklung ein. Sobald die Reaktion begann, wurde trockue Koblensäure eingeleitet und die Temperatur langsam auf $190^{\circ}$ mittels eines Ölbades gesteigert. Zwei Drittel des Metalls gingen ziemlich rasch in Lösung. Nachdem ca. 6 Stunden erwärmt worden war, war noch etwa ein Sechstel des Magnesiums ungelöst. Es wurden noch $5 \mathrm{~g}$ Dimethylanilinjodhydrat zugegeben und dann noch 4 Stunden im 
Koblensäurestrom auf $225^{\circ}$ erhitzt. Fast alles Magnesium verschwand. Am Kolbenhalse setzte sich eine dicke Schicht eines weißen Salzes $a b$, das durch Erwärmen wieder in den Kolben gebracht wurde. Die Reaktionsmasse war nacb dem Erkalten zähflüssig, fast fest und hellbraun gefärbt. Sie wurde in verdünnter Essigsäure und Äther aufgelöst, wobei unter starker kntwicklung von Koblensäure zwei Schichten entstanden. Die obere wurde abgenommen, mit den ätberischen Auszügen der unteren vereinigt und zweimal mit verdünntem Ammoniak ausgezogen. Die ammoniakalische Lösung gab mit verdïnnter Essigsäure einen rein weißen Niederschlag, der gewaschen und getrocknet $2.5 \mathrm{~g}$ wog und sich als reine $p$-Dimethylamino-benzoesäure vom Scbmp. $240^{\circ}$ erwies. Es wares demnach etwa $18.3^{\circ}{ }^{\circ}$ des ursprünglich zugesetzten Jodbydrats in Dimethylaminobenzoesäure übergegangen.

\section{Versuche mit Äthyl-anilin.}

Darstellung des Äthyl-phenyl-carbaminats in Äther.

Tum Vergleich mit dem entsprechenden Verfabren beim Methylcnilin wurde das $\ddot{A}$ thylanilin zunächst ebenfalls mit einer ätherischen lösung von Metbylmagnesiumjodid umgesetzt, und zwar kamen $10.8 \mathrm{~g}$ Naguesiumspäne, $58.7 \mathrm{~g}$ Jodmethyl und $50 \mathrm{~g}$ Äthylanilin zur Anwendung. Fin tertiäres Amin wurde nicht zugesetzt. Nach etwa $1 \dot{j}$-stindigem Erhitzen des Gemisches im Kohlensäurestrom auf etwa $220^{\circ}$ wurde das erkaltete Produkt, eine braune, gummöse Masse, in der üblichen Weise mit Essigsäure und Äther zersetzt und so $1.2 \mathrm{~g}$ einer grauen Aminosäure erbalten. Sie wurde in verdünnter Schwefelsäure gelöst und nit Natriumacetat gefält, sodann mebrfach aus Wasser umkrystallisiert, bis sie den konstanten Schmp. $195^{\circ}$ zeigte. In Essigsäure und Alkohol ist sie leicht löslich, wenig in Benzol und in Wasser, nicht in Petroläther und Ligroin.

Wie die Analysen ergaben, lag hier die

F- ̈̈thylmethylamino-benzoesäure, $\left(\mathrm{C}_{2} \mathrm{H}_{5}\right)\left(\mathrm{CH}_{3}\right) \mathrm{N} . \mathrm{C}_{6} \mathrm{H}_{4} . \mathrm{COOH}$, vor.

0.1431 g Sbst.: $0.3517 \mathrm{~g} \mathrm{CO}_{2}, 0.0966 \mathrm{~g} \mathrm{H}_{2} \mathrm{O} .-0.1640 \mathrm{~g}$ Sbst.: $10.1 \mathrm{ccm}$ N $\left(18^{\circ}, 779.5 \mathrm{~mm}\right)$.

$$
\begin{aligned}
& \mathrm{C}_{10} \mathrm{H}_{13} \mathrm{O}_{2} \mathrm{~N} . \text { Ber. C } 66.98, \mathrm{H} 7.81, \mathrm{~N} 7.84 . \\
& \text { Gef. 》 67.12, 》 7.55, } 7.34 .
\end{aligned}
$$

Die Entstehung der Säure ist offenbar durch überschüssiges ,Jodmetbyl veranlaßt, welches die in erster Linie zu erwartende $p$-Äthylaminobeuzoesäure methyliert. 
$p$-Äthylnitrosamin-benzoesäure, $\left(\mathrm{C}_{2} \mathrm{H}_{5}\right)(\mathrm{NO}) \mathrm{N}$. $\mathrm{C}_{6} \mathrm{H}_{4}$. COOH.

Die Mutterlauge, aus der durch Fällen mit Essigsäure die eben beschriebene tertiäre Aminosäure ausgefällt worden war, konnte noch unmethyliert gebliebene $p-\ddot{A}$ thylamino-benzoesäure enthalten, da die sekundären Aminosïuren der $p$-Reihe bedeutend löslicher sind als die $p$-Dialkylaminobeuzoesäuren. Die Mutterlauge wurde daher mehrfach ausgeäthert und der ätherische Auszug zur Trockne verdampit. Der Rückstand wurde in verdünnter Schwefelsäure gelöst und bei Eiskälte nitrosiert. Es fiel ein Niederschlag in gelben Flocken aus, der getrocknet $2 \mathrm{~g}$ wog. Aus Wasser wiederholt umkrystallisiert, wurde die Nitrosaminsäure in gelben Nadeln vom Schmp. $186^{\circ}$ (Capillar-

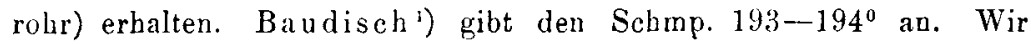
vermuteten daher, die von uns gewounene S̈äure sei noch nicht ganz rein. Die Analysen gaben aber stimmende Zahlen.

0.1033 g Sbst.: $0.2111 \mathrm{~g} \mathrm{CO}_{2}, 0.0498 \mathrm{~g} \mathrm{H}_{2} \mathrm{O} .-0.1122 \mathrm{~g}$ Sbst.: $13.9 \mathrm{ccm}$ $\mathrm{N}\left(20^{\circ}, 75 \mathrm{~g} \mathrm{ccm}\right)$.

$$
\begin{aligned}
& \mathrm{C}_{9} \mathrm{H}_{10} \mathrm{O}_{3} \mathrm{~N}_{2} \text {. Ber. C 55.63, H 5.19, N } 14.16 \text {. } \\
& \text { Gef. »55.81, 》5.39, 》14.25. }
\end{aligned}
$$

Natürlich ist auch bier vicht ausgeschlossen, daß die Nitrosaminsäure aus tertiärer Säure durch Austausch eines Metbyls gegen $\mathrm{Ni}$ trosyl entstanden ist. Man geht aber kaum fehl, wenn man annimmt, dali sie der Hauptsache nach aus sekundärer Süure stanımt.

Darstellung und Umlagerung des Äthyl-phenyl-carbaminats in Diäthylaviliu.

$2.02 \mathrm{~g}$ Magnesium, $12.9 \mathrm{~g}$ Jodäthyl und $20.2 \mathrm{~g}$ Diätbylanilin wurden nit $10 \mathrm{~g}$ Äthylanilin gemischt und langsam in Koblensäurestrom am Steigrohr auf $235^{\circ}$ (Ölbad) erbitz.t. Da sich nicht alles Metall auflöste, wurde nachtrïglich noch ein kleiner Überscbuß von dodäthyl zugesetzt. Nach 15-16-stündigem Erhitzen wurde die Reaktionsmasse in der gewöbnlichen Weise zersetzt. Man erhielt $2.5 \mathrm{~g}$ einer Aminosïure, die sich als die bereits von Michler und Gradmann ${ }^{2}$ ) beschriebene $p$-Diäthylamino-benzoesäure erwies.

Darstelluug und Umlagerung des $\ddot{A}$ thyl-phengl-carbaminats in Ätbylauilin.

Bei diesem Versucb wurde wieder mit Jodmethyl an Stelle des in vorigen Versuch angewendeten Jodäthyls gearbeitet, und $\mathrm{zwar}$ wurden in einen Bombenrohr $20 \mathrm{~g} \ddot{A}$ tbylanilin, $12 \mathrm{~g}$ Jodmethyl und 2.02 g Magnesiamspäne zunächst auf dem Wasserbade zur Reaktion

1) Diese Berichte 39, 4:98 [1906]. 2) Diese Berichte 9, 1912 [1876]. 
gebracht, wobei die Reaktionsmasse vor Feuchtigkeit geschützt wurde. Nach dem Erkalten wurde trockne Kohlensäure eingeleitet, daun das Rohr zugeschmolzen und 20 Stunden lang auf $200-210^{\circ}$ erhitzt. Es war dann eine durchscheinende feste Masse von bräunlicher Farbe entstanden. Die Zersetzung ergab $4.5 \mathrm{~g}$ Aminosäure, die, einige Male aus Wasser umkrystallisiert, den konstanten Schmp. $195^{\circ}$ (Capillarröhrcheu) zeigte und aus $p$ - Athylmetbylamino-benzoesäure bestand, wie die Analysen bestitigten.

$0.1607 \mathrm{~g}$ Sbst.: $0.3957 \mathrm{~g} \mathrm{CO}_{2}, 0.1047 \mathrm{~g} \mathrm{H}_{2} \mathrm{O} .-0.1836 \mathrm{~g}$ Sbst.: 12.1 cem $\mathrm{N}\left(20^{\circ}, 754 \mathrm{~mm}\right)$.

$$
\begin{array}{r}
\mathrm{C}_{10} \mathrm{H}_{13} \mathrm{O}_{2} \mathrm{~N} . \text { Ber. C 66.98, H 7.31, N } 7.84 . \\
\text { Gef. » } 67.15, » 6.71 \text {, } 7.51 .
\end{array}
$$

Hier wie bei allen vorhergehenden Versuchen trat eine beträchtlicbe blaue Fluorescenz der Lösungen der Reaktionsmasse auf, was auf Vorbandensein von o-Amivosäure schließtn ließ. Doch gelang es uns erst später, solche Säuren wirklich nachzuweisen und zu isoliereu. Wir werden darüber noch berichten.

Als Nebenprodukte entstehen, wie bereits erwähnt, in den meisten Fällen Harnstoffe in größerer oder geringerer Menge. Die Entstehung derselben ist im ersten Teil der Arbeit besprochen.

\section{Ad. Grün und A. von Skopnik: Synthese der dreifach-gemischten Glyceride.}

[3. Mitteilung über die Synthese der Fette.] (Eingegangen am 4. Oktober 1909.)

Bekanntlich wurden die Fette lange Zeit für Gemische von sogenannten einfachen Triglyceriden gehalten, und das Problem ibrer Synthese galt als gelöst, nachdem Berthelot $\left.{ }^{1}\right)$ die Darstellung der Verbindungen des Typus $\mathrm{C}_{3} \mathrm{H}_{5}(\mathrm{O} . \mathrm{CO} . \mathrm{R})_{3}$ gezeigt hatte.

In neverer Zeit wurde aber festgestellt, da $\beta$ in der Natur neben diesen einfachen auch ngemischte Glycerides vorkommen und zwar sowohl Triglyceride mit zwei, als auch mit drei verschiedenen Acylen,

1) Chimie org. fondée sur la synthèse, Bd. II 